\title{
Improving the early diagnosis of early nodular melanoma: can we do better?
}

\section{Paola Corneli, Iris Zalaudek, Giovanni Magaton-Rizzi \& Nicola di Meo}

To cite this article: Paola Corneli, Iris Zalaudek, Giovanni Magaton-Rizzi \& Nicola di Meo (2018): Improving the early diagnosis of early nodular melanoma: can we do better?, Expert Review of Anticancer Therapy, DOI: 10.1080/14737140.2018.1507822

To link to this article: https://doi.org/10.1080/14737140.2018.1507822

Accepted author version posted online: 06 Aug 2018.

Submit your article to this journal $₫$

View Crossmark data $\nearrow$ 
Review

Improving the early diagnosis of early nodular melanoma: can we do better?

Paola Corneli, Iris Zalaudek*, Giovanni Magaton-Rizzi and Nicola di Meo

Dermatology Clinic, Maggiore Hospital, University of Trieste, Italy

${ }^{*}$ Corresponding author:

Prof Iris Zaludek

Head of Dermatology Clinic, Maggiore Hospital,

University of Trieste, IV Piano Palazzina Infettivi,

Piazza Ospitale 1, 34100 Trieste Italy

Email: iris.zalaudek@gmail.com 


\section{Abstract}

Introduction: Cutaneous melanoma is the 6th most common malignant cancer in the USA. Among different subtypes of melanoma, nodular melanoma (NM) accounts about $14 \%$ of all cases but is responsible for more than $40 \%$ of melanoma deaths. Early diagnosis is the best method to improve melanoma prognosis. Unfortunately, early diagnosis of NM is particularly challenging given that patients often lack identifiable risk factors such as many moles or freckles. Moreover, early NM may mimic a range of benign skin lesions that are not routinely excised or biopsied in every day practice. For this reason, specific clinical and skin imaging clues have been proposed to improve early detection of NM.

Areas covered: The review discusses about the noninvasive tools to diagnose thin melanoma, particularly NM.

Expert commentary: Currently dermatologists present a wide opportunity of diagnostic tools. Current data suggest that the early diagnosis of NM is a major challenge as the majority of early NM are symmetric, roundish and lack specific pattern. Another promising strategy is based on recent data suggesting that artificial intelligence based on deep convolutional neural networking (CNN) is able to outperform average dermatologist. Further research is necessary to validate the performance of this method in the real world and in the clinical setting.

Keywords: nodular melanoma, dermoscopy, reflectance confocal microscopy, noninvasive imaging tools, skin cancer, dermato-oncology. 


\section{Introduction}

Cutaneous melanoma (CM) is the 6th most common malignant cancer in the USA. The term CM encompasses a heterogeneous subset of malignant melanocytic proliferations that differ significantly respect to their epidemiology, morphology, growth dynamics, genetics and potential to metastasize. Currently, 3 main groups are distinguished, slow growing melanoma (superficial spreading melanoma), fast growing melanoma (nodular subtype) and classical lentigo maligna melanoma. Among various subtypes of $\mathrm{CM}$, nodular melanoma (NM) accounts for $14 \%$ of all CM but is responsible for more than $40 \%$ of melanoma deaths [1]. This type of melanoma is characterized by a rapid growth rate and the estimated invasion rate of NM is about $0.5-\mathrm{mm}$ per months [2]. Regardless of the striking differences between these three main groups of melanoma, many still agree with the traditional unifying model of melanoma progression, which postulates that all melanomas derive from malignant transformed epidermal melanocytes, which initially proliferate along the basal layer before acquiring the capability to invade the dermis. Because of epidemiological, morphological and genetical differences between NM and other subtypes of melanoma, some postulate that NM may origin in the dermis rather than in the epidermis and just secondarily will give rise to clinical recognizable features on the skin $[3,4]$. Data report that about $40-50 \%$ of NM will have a tumor thickness of $2+\mathrm{mm}$ at time of diagnosis, justifying the hidden intradermal growth that makes clinical visible the lesion only at an advanced phase $[4,5]$. More than $40 \%$ of melanoma deaths are attributed to this aggressive form of melanoma [6,7], this underlines the need for improving the early diagnosis of NM. However, this goal is hampered by some challenging characteristics of this melanoma subtype. In this manuscript we will provide a practically oriented overview of the current literature focusing on strategies to improve early NM diagnosis. 


\section{Epidemiology}

Epidemiological data suggest that fast growing NM more commonly affects men, aged 50+ years, who lack any of the known melanoma risk factors established for other forms of CM such as multiple nevi, freckles or signs of sun-damage [6-8]. Notably, the group of people with the highest risk for NM, are also constantly under-represented during skin cancer screening programs [9]. On the other hand, screening programs seem to have little impact on the prompt diagnosis of early NM, as the majority of tumors develop rapidly de novo on previously unaffected skin and are mainly patient detected [10]. According to patients' interviews, the histories of their first taking notice of the tumor are described as "suddenly appearing" or "bulging out" of healthy skin $[11,12]$. Whether or not, regular skin cancer screening examinations will allow for an earlier diagnosis compared to patient selfdetection remains currently questionable; as a matter of fact, we cannot predict when or where these melanomas will develop and given their rapid growth (estimated about 0.5$\mathrm{mm}$ per month [2]), regular skin examinations of at least every 2 months would be required for their early detection. Thus, population-based strategies containing images and information about the clinical features of NM may represent better strategies to improve the knowledge and recognition about this aggressive subtype of $\mathrm{CM}$ in a given population.

\section{Clinical features and clues}

Another challenging problem with early NM diagnosis is related to its peculiar clinical appearance. Several lines of evidence suggest that the majority of tumors appear as clinically symmetric red to pink or gray-blue plaques or nodules with a diameter $<6 \mathrm{~mm}$ (Fig.1-2) $[5,13,14]$. Accordingly, early NM escape the so-called ABCD rule (asymmetry, border irregularity, color variegation and diameter greater than $6 \mathrm{~mm}$ ) summarizing the key features of superficial spreading melanoma [15]. For this reason, alternative rules, named the EFG and/or CCC rule have been proposed in the clinical diagnosis of NM [15, 16]. The 
former EFG summarizes the characteristic features of NM, namely elevation, firmness on palpation and continuous growth for more than 1 month [15], while the latter $3 \mathrm{C}$ stand for color (uniform red to blue), contour (roundish) and change (rapid growth) [16]. Another problem related to its clinical features is the fact that it may conceal common benign lesions such as hemangiomas, intradermal nevi or seborrheic keratosis [17, 18], which are not routinely biopsied as they are usually not included in the differential diagnosis of melanoma. As the latter are commonly stable lesions, the history of sudden development/evolution and fast growth appear the most important criteria in the diagnosis of NM. Nevertheless, a high level of suspicion is required to recognize these early forms of NM. For this reason, if after an accurate and careful clinical examination a NM cannot be ruled out, immediate excision should be prompted [19].

\section{Non-invasive skin imaging tools}

The introduction of non-invasive diagnostic tools such as dermoscopy and later, reflectance confocal microscopy (RCM) improved significantly the clinicians' diagnostic accuracy for melanoma detection $[20,21]$. This is because they permit the visualization of sub-macroscopic structures invisible for the naked eye [21]. Furthermore, there are more recently employed methods in the non-invasive diagnosis of melanoma such as optical coherence spectroscopy (OCT) and electrical impedence spectrocopy (EIS), not routinely used [22]. However, with the exception of dermoscopy allowing a fast and reliable examination of almost all skin lesions, all other methods are limited to clinically selected lesions, thus representing second level diagnostic tools for clinically and dermoscopically selected equivocal lesions.

\subsection{Dermoscopy of nodular melanoma}

Dermoscopy is a practical and simple non-invasive diagnostic tool that allows having an improved diagnosis of skin cancer, particularly pigmented ones. It is based on an optical 
instrument called dermatoscope that allows to observe sub-macroscopic patterns of skin lesions not visible to naked eye. As most dermoscopic features correlate to specific underlying histopathologic correlates, it aids clinicians to better predict the histopathological diagnosis of a given skin tumor [23]. The dermatoscope is equipped with a lens and through a light, able to cross the stratum corneum, allows for the observation of cells down to the dermo-epidermal junction and papillary dermis.

Various dermoscopic criteria such as asymmetry in colors and structures, atypical pigmented network, irregular streaks, irregular brown globules, irregular brown structures areas and regression features are used to diagnose melanoma, especially superficial spreading subtype [24]. Instead, NM often lacks these patterns and represents a challenging diagnosis even when examined by dermoscopy. However, there are some criteria that should raise the index of suspicion for NM:

1. Pigmented NMs often lacks the aforementioned features but could be dermoscopically recognized by the presence of blue and black colors, defined as a combination of structureless blue areas, black dots/globules, and blotches, involving at least $10 \%$ of the lesion surface. The so-called blue-black rule has enhanced the discrimination of NMs from other benign nodular tumors, such as hemangioma, angiokeratoma, blue nevus or seborrheic keratosis [25]. Although blue color is seen in these benign lesions, the combination together with black dots and blotches is rarely present in benign skin lesions. Blue under dermoscopy has been correlated to the presence of melanin in the deep dermis, while black color is associated with both, epidermal melanin or hemoglobin in the stratum corneum, but also with dermal aggregates of pigmented melanocytes under an attenuated, thinned epidermis. For this latter reason black color may be even predictive for a high-risk ulceration [26]. For changing heavily pigmented skin lesions, the so-called BB rule represents a highly effective and important clue for the diagnosis of pigmented NM (Fig.3) [25]. 
2. It is well documented that NM often lacks pigmentation (Fig.4) [27]. In case of amelanotic or hypomelanotic NM (AHNM) the diagnosis does not rely on pigmented but vascular patterns [28]. While true amelanotic melanoma lacks any pigmentation, AHNM is characterized by residual pigmentation or light pigmentation [29]. In the latter forms, the presence of brown, gray or blue pigment, especially when seen on the base of a pink nodule, are helpful criteria to raise the suspect of melanoma. In cases of amelanotic melanoma, milky-red areas, short white shiny streaks (only seen under polarized dermoscopy) and polymorphous vascular pattern are the only clues for the diagnosis [27, 28]. Polymorphous vessels are defined as more than one morphological type of vessel; the most frequent combination of vessel types seen in melanoma is linear, coiled and dotted. Notably, in contrast to basal cell carcinoma or well-differentiated squamous cell carcinoma, which are characterized by evident larger size vessels, NM more frequently exhibits microvessels of small diameter and length (personal observation). Milky red globules are defined as unfocused large ovoid or polygonal structures of pink-white color, often showing a central linear irregular or corkscrew vessel. This latter probably represents neoangiogenesis [28]. It has been shown that thin AHNM is more difficult to diagnose than thick one, as in thin tumors vessels the vascular polymorphism is less evident [28-30]. Short, shiny white streaks (also known as white thick lines, chrysalis or crystalline structures) are seen only under polarized dermoscopy, but represent an important criterion for the diagnosis, especially when associated with polymorphic vessels or pink areas [31]. Although short, shiny white streaks are not very specific for the diagnosis of NM, it is only rarely seen in benign skin tumors, thus representing an important management criterion [32] 
The clinical diagnosis of NM remains challenging even if coupled with dermoscopy. For this reason, specific management rules have been introduced, combining clinical and dermoscopic criteria [33].

1. Any nodular lesion that cannot be confidently diagnosed as benign lesion requires immediate excision. Dermoscopic follow-up of doubtful nodular lesion is strongly discouraged, because in the case of NM, it might worsen the prognosis [19].

2. Pyogenic granuloma and NM may reveal overlapping clinical and dermoscopic criteria that do not allow differentiating between both entities. Therefore, the diagnosis of pyogenic granuloma should always be confirmed by histopathology, irrespective of the age of the patient. The latter statement is based on the fact that atypical spitzoid tumors, often affecting children, are also included in the differential diagnosis of pyogenic granuloma. Destructive methods such laser therapy and/or liquid nitrogen are strongly discouraged as they destroy tissue without the possibility of a histopathological examination [34]

3. Pink lesions with the suspect of a spitzoid neoplasm should be always excised, irrespective of age of the patient [34-7].

4. Any ulcerated non-pigmented nodule should be excised [19].

5. Any lesion showing polymorphous vessels should be excised [38].

6. Pink lesions, for which dermoscopy does not confirm the clinical suspected diagnosis, should be dealt with caution [19].

7. Blue nodules, lacking a convincing subjective history of a stable lesion a/o showing dermoscopically blue and black areas, should be excised [25]

\subsection{Reflectance Confocal Microscopy in nodular melanoma}

Reflectance Confocal Microscopy ( $\mathrm{RCM})$ is a non-invasive diagnostic tool that allows the observation of structures at a quasi-histopathologic resolution. For this reason, RCM is 
considered by some as virtual biopsy. RCM uses a diode as a monochromatic light that is able to penetrate the skin thickness. This light is reflected and generates an image in the detector on the base of the different refractive index of the tissue [22].

Histologically, NMs are characterized by vertical growth without evidence of an associated radial growth beyond the width of three rete ridges beyond the invasive component [39]. RCM has contributed significant improvement in the early diagnosis of NMs. Some authors proposed a semi-quantitative algorithm for RCM evaluation of melanocytic lesions. They include: two major criteria, presence of non-edged papilla and cytologic atypia; four minor criteria, presence of roundish cells in the superficial layers, pagetoid cells widespread throughout the lesion, cerebriform clusters and nucleated cells within the dermal papilla [40]. NM lacks some confocal features of the superficial spreading melanoma, such as epidermal disarrangement and pagetoid spreading. Massive proliferation in the dermis causes missing of the typical papillary architecture. Basal layer and upper dermis show pleomorphic cells with bright cytoplasm and dark nuclei, whereas amorphous, hyporefractive nest called "cerebriform nest" are found in the deep dermis. This latter is a relevant criterion for the diagnosis of NM [20]. Other authors correlate dermoscopic BB feature with confocal features in NM [21]. Dermoscopic black color is associated with two different patterns: large black blotches and irregular black dots/globules. Black blotches result from the epidermis being totally filled by upward-migration melanocytes as nests and pagetoid cell, whereas black dots/globules correspond to the epidermis having spared areas between the upward-migrating nests and pagetoid cells. Black color results not only from epidermal melanin or hemoglobin (in case of ulceration), but also from a dense dermal proliferation of pigmented melanocytes under a significantly thinned, yet not ulcerated epidermis. Accordingly, black color may be even predictive of ulceration before it took place [26], which is a known, negative prognostic criterion. The dermoscopic criterion 
of short shiny white streaks appears to correlate dermal fibrosis or collagen bundles under $\operatorname{RCM}[20,41]$.

Furthermore, RCM has been demonstrated to be an add-on diagnostic tool especially in the context of amelanotic/hypopigmented melanocytic tumors, because of the high refractive index of melanocytes that produce visible reflections upon the $\mathrm{RCM}$ examination, allowing for an improved recognition and differentiation between these difficult to diagnose lesions. Therefore, combination of dermoscopy and RCM represents a valuable method to detect AHNM [42]

\section{Other Non-invasive Methods}

Besides dermoscopy and RCM, which are evidence proven methods for improving the melanoma diagnosis, more recent new non-invasive diagnostic tools have been introduced to aid the early detection of melanoma. These include optical coherence spectroscopy (OCT) and electrical impedence spectrocopy (EIS) [22]. However, these instruments are limited to specialized centers and not yet implemented in the daily routine.

\subsection{Optical Coherence Tomography (OCT)}

OCT is a non-invasive diagnostic tool, first developed in ophthalmology, but is now also used in dermatology $[22,43]$ OCT is an interferometric procedure, able to detect the intensity of backscattered infrared light from biological tissue by measuring the optical path length [43]. Different OCT methods are studied: Frequency Domain OCT (FD-OCT), Dynamic OCT (D-OCT), High Definition OCT (HD-OCT). FD-OCT is primarily used in the diagnosis of basal cell carcinoma and other non-pigmented skin cancers. Although there are some reports on the OCT patterns of melanoma, [43-47] further studies are needed to understand the real role of OCT in the early diagnosis of NM. 


\subsection{Electrical Impedence Spectrocopy (EIS)}

Electrical impedence spectroscopy (EIS) is a novel not visual method employed in skin cancer diagnosis. This method does not make a diagnosis but suggests based on scores whether a lesion should be considered benign or malignant. EIS relieves the current that flows between the cells. Tumor cells are characterized by irregular curves due to their polymorphism. From these curves, the system calculates a score that reflects the degree of abnormality of the lesion. In a pivotal trail [47], EIS has proved to achieve a high sensitivity (96.6\%) without fully compromising the specificity $(34,4 \%)$ however, the system is limited to flat tumors and therefore further studies are needed for assessing its role in the diagnosis of NM [22].

\section{Expert commentary}

Currently, dermatologists present a wide opportunity of diagnostic tools, that includes the aforementioned dermoscopy, RCM, OCT and EIS. Other non-invasive diagnostic tools include dermatofluoroscopy, optoacoustic imaging and other diagnostic tools not validated for the diagnosis of pigmented lesions, nor for NM. [22]

Current data suggest that the early diagnosis of NM is a major challenge as the majority of early NM are symmetric, roundish and lack specific pattern. Moreover, even if diagnosed early, the majority of tumors reached already an invasion thickness of 2-mm, for this reason known as fast growing melanoma. As most NMs are patient self-detected, population-based interventions should aim to make the population aware about the key features of this aggressive melanoma subtype, namely a suddenly appearing and fast growing pink or blue nodule.

Another promising strategy is based on recent data suggesting that artificial intelligence based on deep convolutional neural networking $(\mathrm{CNN})$ is able to outperform average dermatologist. CNN is able to classify skin lesions using only pixels and disease labels as 
inputs. This fast method could be used on mobile device and has the potential to increase the decision-making power in clinical practice. Further research is necessary to validate the performance of this method in the real world and in the clinical setting [48].

Other possibility is the use of smartphone application. Currently the list of available apps are able to provide educational materials, photographic atlas and skin self-examination tutorial. These apps allow the self-surveillance and through digital images they detect changing and new moles during their self-examination. Some apps allow risk class stratification of the pigmented lesion in high, medium and low risk by using pattern recognition software comparing a database of nevi with confirmed melanoma diagnoses. The problem is the risk of misclassified lesions, mostly for NM [49]. The use of smartphone and internet have introduced teledermatology, a way to transmit medical information to a dermatologist for remote evaluation. Medical information includes clinical history, macroscopic and dermoscopic photos, if possible [50]. Teledermoscopy is a rapidly evolving method that follows a direct patient care model by enabling patient to initiate a consultation with a dermatologist. The consultation in teledermoscopy is store-andforward: patient's medical information is stored and then reviewed by a medical provider [50]. The expected benefits are an accurate risk indication, immediate response of dermatologist and finding skin cancer early. Furthermore, teledermoscopy could partially resolve the problem of accessing to a specialist. Limitations are the inability to complete full-body examination, particularly for genital area of the body and the risk of misdiagnosis. [50] Some authors demonstrate that these applications have a sensitivity for melanoma of $73 \%$ and specificity of $83 \%$, whereas dermatologists have sensitivity of $88 \%$ and specificity of $97 \%$. [51] Other authors demonstrate that smartphone apps have scarce scientific evidence, studies are small and have low methodological quality and only one-third of the app has assessed diagnostic accuracy [52]. Thus, new apps may come to the market, which may significantly aid the diagnosis of early NM. 


\section{Five-year view}

Early NM diagnosis is a problem related to its peculiar clinical appearance and rapid growth rate. While regular screening programs are unlikely to detect NM at early stage, population-based strategies aiming to increase the knowledge and awareness about risk factors and clinical features associated with fast growing NM seem more promising attempts to allow for an earlier detection of NM. Particularly the development of artificial intelligence in skin cancer detection may play a crucial role in both, population-based information and self-detection of aggressive forms of melanoma.

\section{Key issues}

- NM does not follow the classic melanoma growth but presents an early invasiveness and causes a high percentage of deaths due to melanoma.

- Its clinical diagnosis is complicated by its unusual clinical appearance, which often does not present the classic characters of melanoma and sometimes mimics benign lesions

- Classical skin cancer screening campaigns are unlikely to improve early diagnosis of NM.

- Future interventions should target on key features such as a rapidly growing blue or red nodule on a population-based level in order to increase the self-detection

- Clinical and dermoscopic and RCM features along with specific management rules have been introduced in order to correctly miss difficult to diagnose NM. 
- Further studies on methods using artificial intelligence may contribute to further improvements in the diagnosis of early NM.

\section{Funding}

This paper was not funded.

\section{Declaration of interests}

The authors have no relevant affiliations or financial involvement with any organization or entity with a financial interest in or financial conflict with the subject matter or materials discussed in the manuscript. This includes employment, consultancies, honoraria, stock ownership or options, expert testimony, grants or patents received or pending, or royalties.

\section{Reviewer disclosures}

Peer reviewers on this manuscript have no relevant financial or other relationships to disclose. 


\section{References}

\section{Reference annotations}

${ }^{*}$ Of interest

** Of considerable interest

1. Mar V, Roberts H, Wolfe R, et al. Nodular melanoma:A distinct clinical entity and the largest contributor to melanoma deaths in Victoria, Australia. J Am Acad Dermatol. 2013:68(4):568-75

2. Liu W, Dowling JP, Murray WK, et al. Rate of Growth in Melanomas. Arch. Dermatol. 2006; 142(12):1551-8.

3. Zalaudek I, Marghoob AA, Scope A, et al. Three roots of melanoma. Arch. Dermatol.2008; 144(10): 1375-9.

4. Zalaudek I, Moscarella E, Longo C, et al. No one should die of melanoma: a vision or impossible mission. Melanoma Manage. 2014; 1(1): 41-6

5. Kalkhoran S, Milne O, Zalaudek I, et al. Historical, Clinical, and Dermoscopic Characteristics of Thin Nodular Melanoma. Arch. Dermatol. 2010; 146(3):311-8.

** A review summarizing historical, clinical and dermoscopic features of early nodular melanomas

6. Chamberlain AJ, Fritschi L, Giles GG, et al. Nodular type and older age as the most significant associations of thick melanoma in Victoria, Australia. Arch. Dermatol. 2002; 138(5):609-14.

7. Murray CS, Stockton DL, Doherty VR. Thick melanoma: the challenge persists. Br. J. Dermatol. 2005;152(1):104-9..

8. Lipsker D, Engel F, Cribier B, et al. Trends in melanoma epidemiology suggest three different types of melanoma. Br. J. Dermatol, 2007; 157(2):338-43.

9. Hübner J, Waldmann A, Geller AC et al. Interval cancers after skin cancer screening: incidence, tumour characteristics and risk factors for cutaneous melanoma. Br. J. Cancer. 2017; 116(2): 253-59.

10. Halpern AC, Marchetti MA, Marghoob AA, et al. Melanoma Surveillance in 'High-Risk' Individuals. JAMA Dermatology. 2014;150(8):815.

11. Chamberlain AJ, Fritschi L, Kelly JW. et al. Nodular melanoma: patients' perceptions of presenting features and implications for earlier detection. J. Am. Acad. Dermatol. 2003; 48(5): 694-701.

12. Warycha MA, Christos PJ, Mazumdar M, et al. Changes in the presentation of nodular and superficial spreading melanomas over 35 years. Cancer. 2008; 113(12): 3341-8.

13. Pizzichetta MA, Kittler H, Stanganelli I et al. Pigmented nodular melanoma: the predictive value of dermoscopic features using multivariate analysis. Br. J. Dermatol. 2015; 173(1):10614.

14. Argenziano G, Cerroni L, Zalaudek I, et al, Accuracy in melanoma detection: A 10-year multicenter survey. J. Am Acad. Dermatol. 2012; 67(1):54-9.

15. Kelly JW, Chamberlain AJ, Staples MP, et al. Nodular melanoma. No longer as simple as ABCD. Aust. Fam. Physician. 2003; 32(9):706-9. 16. Moynihan GD. The 3 Cs of melanoma: time for a change?. J. Am. Acad. Dermatol. 1994; 30(3):510-1..

17. Carrera C, Segura S, Palou J, et al. Seborrheic keratosislike melanoma with folliculotropism. Arch. Dermatol. 2007; 143(3):373-6.

18. Carrera C, Marghoob AA. Discriminating Nevi from Melanomas: Clues and Pitfalls. Dermatol. Clin. 2016; 34(4):395-409.

19. Moscarella E, Lallas A, Longo C, et al. Performance of the 'if in doubt, cut it out' rule for the management of nodular melanoma. Dermatol. Pract. Concept. 2017;7(3): 1-5. 
** An abstract presenting the correct management for nodular lesions

20. Segura S, Pellacani G, Puig S, et al. In Vivo Microscopic Features of Nodular Melanomas. Arch. Dermatol. 2008; 144(10): 1311-20 .

21. Longo $\mathrm{C}$, Farnetani $\mathrm{F}$, Ciardo $\mathrm{S}$, et al. Is confocal microscopy a valuable tool in diagnosing nodular lesions? A study of 140 cases. Br. J. Dermatol. 2013; 169(1):58-67.

22. Welzel J, Schuh S. Noninvasive diagnosis in dermatology. JDDG J. der Dtsch. Dermatologischen Gesellschaft. 2017; 15(10):999-1016.

23. Yadav S, Vossaert KA, Kopf AW, et al., Histopathologic correlates of structures seen on dermoscopy (epiluminescence microscopy). Am. J. Dermatopathol. 1993; 15(4):297-305.

24. Walter FM, Prevost AT, Vasconcelos J, et al. Using the 7-point checklist as a diagnostic aid for pigmented skin lesions in general practice: a diagnostic validation study. Br. J. Gen. Pract. 2013; 6(610):343-53.

25. Argenziano $\mathrm{G}$, Longo $\mathrm{C}$, Cameron A, et al., Blue-black rule: a simple dermoscopic clue to recognize pigmented nodular melanoma. Br. J. Dermatol. 2011; 165(6):1251-55.

* An abstract presenting the rules of dermoscopy and the importance of blue black colours for pigmented nodular melanoma

26. Longo C, Farnetani F, Moscarella E, et al. Can noninvasive imaging tools potentially predict the risk of ulceration in invasive melanomas showing blue and black colors?. Melanoma Res. 2013; 23(2): 125-31.

** A review presenting the use of non-invasive diagnostic tools and the rules of dermoscopy and reflectance confocal microscopy

27. Pizzichetta MA, Kittler H, Stanganelli I, et al. Dermoscopic diagnosis of amelanotic/hypomelanotic melanoma. Br. J. Dermatol. 2017; 177(2):538-40.

28. Zalaudek I, Kreusch J, Giacomel J, et al. How to diagnose nonpigmented skin tumors: A review of vascular structures seen with dermoscopy. J. Am. Acad. Dermatol. 2010; 63(3):377-86..

* A review reporting the importance of dermoscopic vascular pattern particularly for amelanotic nodular melanoma.

29. Menzies SW, Moloney FJ, Byth K, et al. Dermoscopic Evaluation of Amelanotic and Hypomelanotic Melanoma. Arch. Dermatol. 2008; 144(9): 1120-7.

30. Pizzichetta MA, Massi D, Mandalà M, et al. Clinicopathological predictors of recurrence in nodular and superficial spreading cutaneous melanoma: a multivariate analysis of 214 cases. J. Transl. Med. 2017; 15(1):227.

31. Di Stefani A, Campbell TM, Malvehy J, et al. Shiny white streaks: An additional dermoscopic finding in melanomas viewed using contact polarised dermoscopy. Australas. J. Dermatol. 2010; 51(4):295-8.

32. Navarrete-Dechent C, Bajaj S, Marchetti MA, et al. Association of Shiny White Blotches and Strands With Nonpigmented Basal Cell Carcinoma: Evaluation of an Additional Dermoscopic Diagnostic Criterion. JAMA dermatology. 2016; 152(5): 546-52.

33. Lallas A, Zalaudek I, Apalla Z, et al. Management Rules to Detect Melanoma. Dermatology. 2013; 226(1):52-60.

34. Ferrari A, Bisogno G, Cecchetto G, et al. Cutaneous melanoma in children and adolescents: The Italian Rare Tumors in Pediatric Age Project Experience. 2014; 164(2): 376-82.

35. Lallas A, Apalla Z, Ioannides D, et al. Update on dermoscopy of Spitz/Reed naevi and menagment guidelines by the International Dermoscopy Sociaty. Br J Dermatol. 2017 $177: 645-55$

36. Brunetti B, Nino M, Sammarco E, et al. Spitz naevus: a proposal for management. J. Eur. Acad. Dermatol. Venereol. 2005; 19(3): 391-3.

37. Dika E, Ravaioli GM, Fanti PA, et al. Spitz Nevi and Other Spitzoid Neoplasms in Children: Overview of Incidence Data and Diagnostic Criteria. Pediatr. Dermatol. 2017; 34(1): 25-32..

38. Zalaudek I, Kreusch J, Giacomel J, et al. How to diagnose nonpigmented skin tumors: A review of vascular structures seen with dermoscopy. J. Am. Acad. Dermatol. 2010; 
63(3):361-74.

39. Clark WH, From L, Bernardino EA, et al. The histogenesis and biologic behavior of primary human malignant melanomas of the skin. Cancer Res. 1969; 29(3):705-27.

40. Pellacani G, Cesinaro AM, Seidenari S, et al. Reflectance-mode confocal microscopy of pigmented skin lesions improvement in melanoma diagnostic specificity. J Am Acad Dermatol. 2005; 53(6):979-85

41. Pellacani G, Bassoli S, Longo C, et al. Diving into the blue: in vivo microscopic characterization of the dermoscopic blue hue. J. Am. Acad. Dermatol. 2007; 57(1): 96-104.

42. Guitera P, Menzies SW, Argenziano G, et al. Dermoscopy and in vivo confocal microscopy are complementary techniques for diagnosis of difficult amelanotic and light-coloured skin lesions. Br J Dermatol. 2016;175(6):1311-1319.

43. Ulrich M, Themstrup L, de Carvalho N, et al. Dynamic Optical Coherence Tomography in Dermatology. Dermatology. 2016; 232(3): 298-311.

44. Gambichler T, Schmid-Wendtner MH, Plura I, et al. A multicentre pilot study investigating high-definition optical coherence tomography in the differentiation of cutaneous melanoma and melanocytic naevi. J. Eur. Acad. Dermatology Venereol. 2015; 29(3):537-54.

45. Cheng HM, Lo S, Scolyer R, et al. Accuracy of optical coherence tomography for the diagnosis of superficial basal cell carcinoma: a prospective, consecutive, cohort study of 168 cases. Br. J. Dermatol. 2016;175(6):1290-300..

46. Gambichler T, Plura I, Schmid-Wendtner M, et al. High-definition optical coherence tomography of melanocytic skin lesions. J. Biophotonics. 2015; 8(8):681-6.

47. Malvehy J, Hauschild A, Curiel-Lewandrowski C, et al. Clinical performance of the Nevisense system in cutaneous melanoma detection: an international, multicentre, prospective and blinded clinical trial on efficacy and safety. Br. J. Dermatol. 2014; 171(5):1099-107.

48. Esteva A, Kuprel B, Novoa RA, et al. Dermatologist-level classification of skin cancer with deep neural networks. Nature. 2017; 542(7639): 115-8.

49. Buller DB, Berwick M, Lantz K, et al. Smartphone Mobile Application Delivering Personalized, Real-Time Sun Protection Advice. JAMA Dermatology. 2015; 151(5):497.

50. Walocko FM, T. Tejasvi T. Teledermatology Applications in Skin Cancer Diagnosis, Dermatol. Clin. 2017;35(4):559-63.

51. Maier T, Kulichova D, Schotten K, et al. Accuracy of a smartphone application using fractal image analysis of pigmented moles compared to clinical diagnosis and histological result. J. Eur. Acad. Dermatol. Venereol. 2015; 29(4):663-7.

52. Buechi R, Faes L, Bachmann LM, et al. Evidence assessing the diagnostic performance of medical smartphone apps: a systematic review and exploratory meta-analysis. BMJ Open. 2017; 7(12): e018280. 


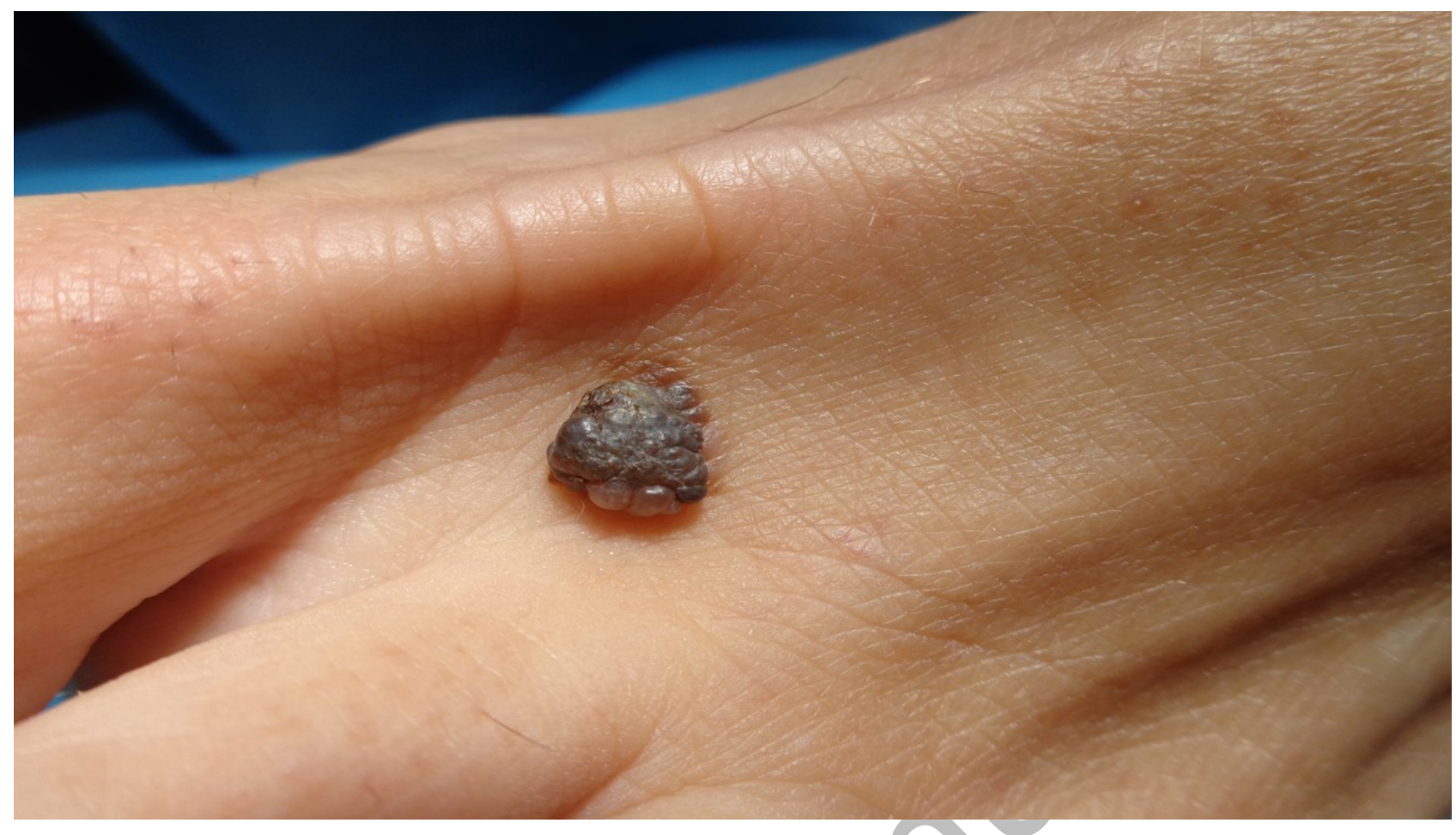

Figure 1: Pigmented NM localized on the dorsum of the foot 


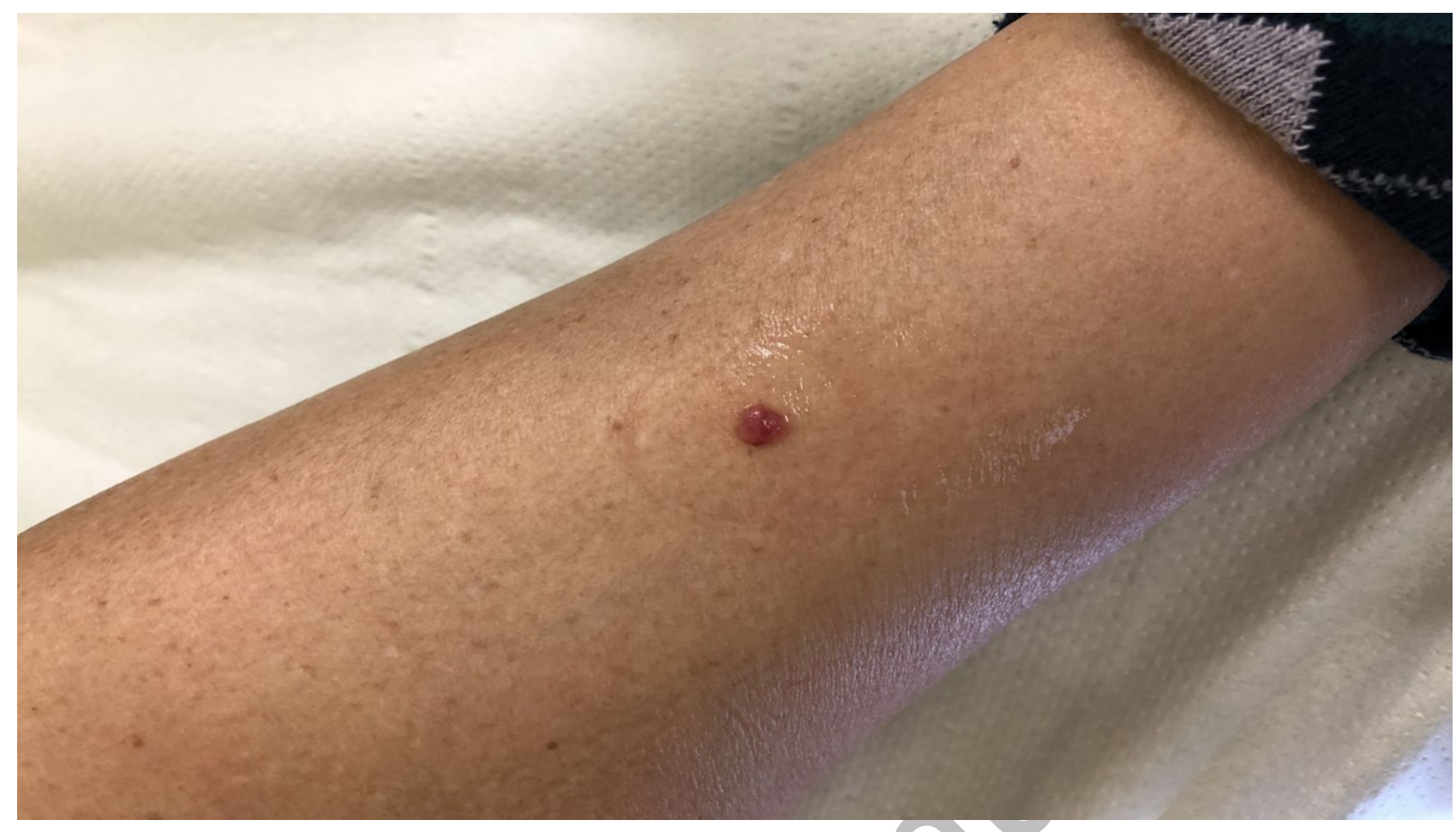

Figure 2: Hypomelanotic NM localized on the lower limb

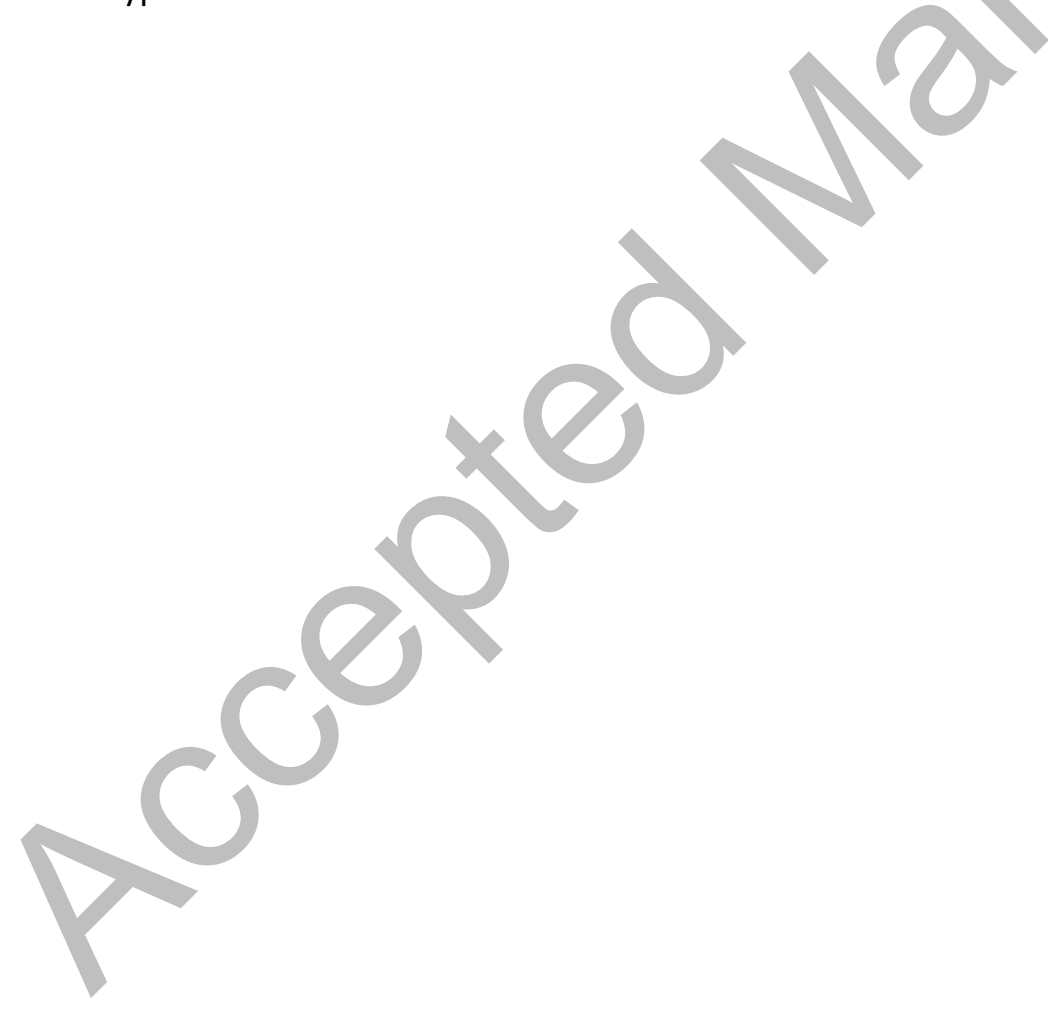




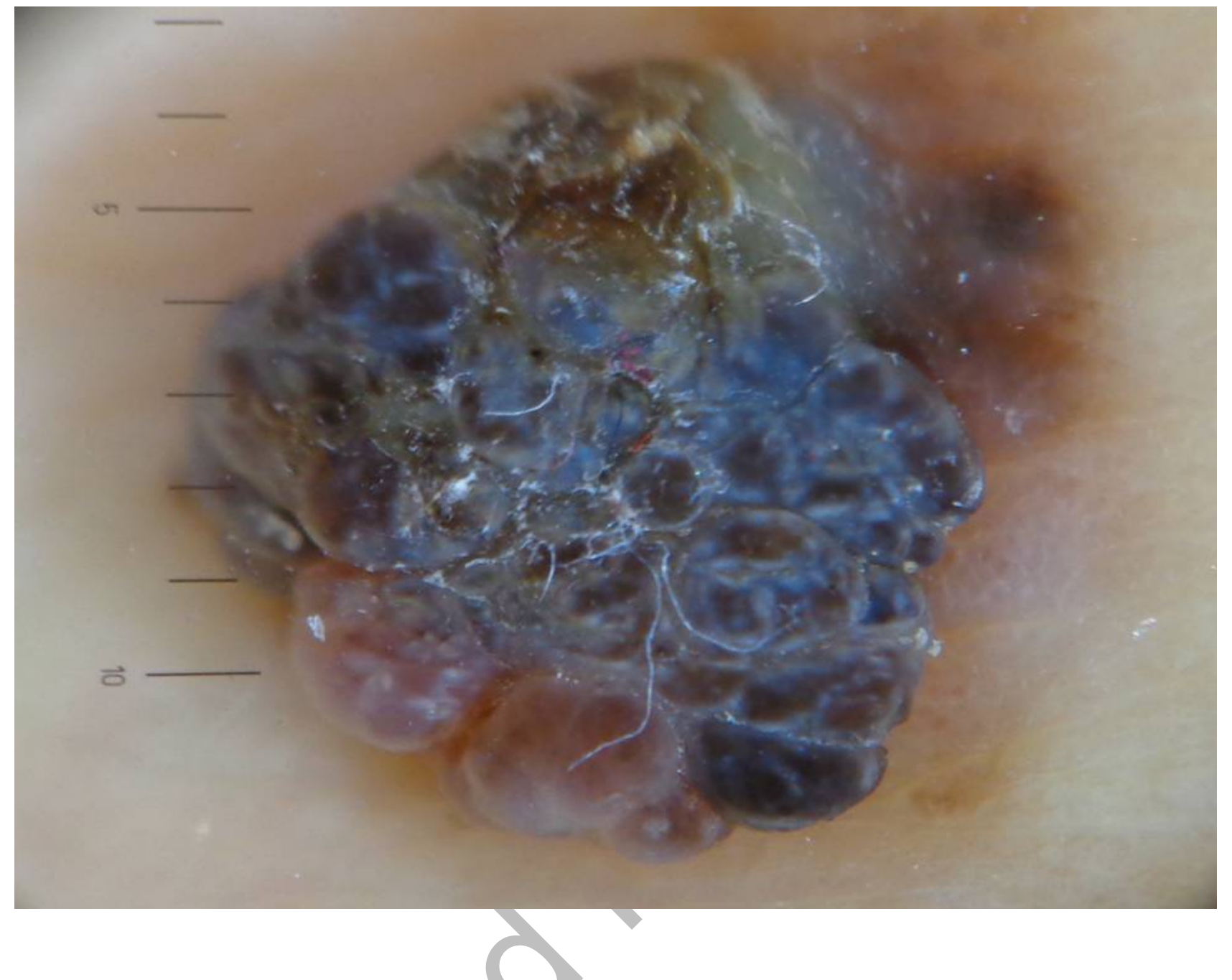

Figure 3: Dermoscopy of pigmented NM of fig.1: black-blue positive NM without standard melanoma criteria 


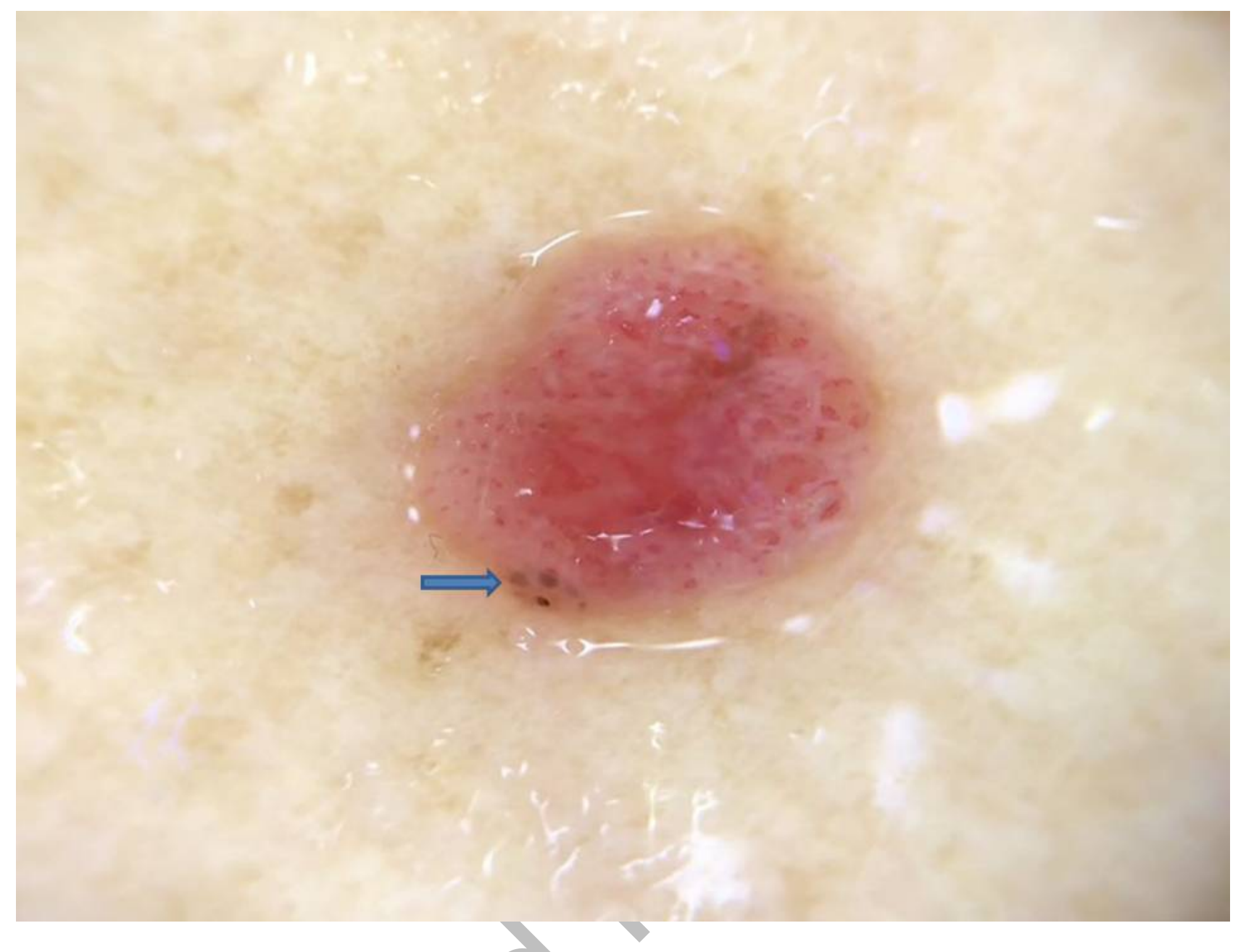

Figure 4: Dermoscopy of hypomelanotic NM of fig.2: polymorphous vascular pattern and some brown globules (blue arrow) 